\title{
ERROS NO PREPARO E NA ADMINISTRAÇÃO DE MEDICAMENTOS INTRAVENOSOS
}

\author{
ERRORS IN THE PREPARATION AND \\ ADMINISTRATION OF INTRAVENOUS DRUGS
}

\section{ERRORES EN LA PREPARACIÓN Y ADMINISTRACIÓN DE MEDICAMENTOS INTRAVENOSOS}

\author{
Ubiane Oiticica Porto Reis ${ }^{1}$ \\ Silvia da Silva Santos Passos ${ }^{2}$ \\ Luciano Marques Santos ${ }^{3}$ \\ Marcelo Silva Reis ${ }^{4}$ \\ Jamille Sampaio Berhends ${ }^{5}$ \\ Carolina Madeiro Meira ${ }^{6}$
}

Como citar esse artigo: Reis UOP, Passos SSS, Santos LM, Reis MS, Berhends JS, Meira CM. Erros no preparo e na administração de medicamentos intravenosos. Rev baiana enferm. 2020;34:e36450.

Objetivo: identificar os erros no preparo e na administração de medicamentos intravenosos. Método: estudo observacional e descritivo. Foram observadas 694 doses de medicamentos intravenosos realizadas pelos profissionais de enfermagem em pacientes adultos nas unidades de Emergência, Internamento e Unidade de Terapia Intensiva de um hospital de pequeno porte do recôncavo da Bahia, Brasil. Os dados foram coletados em março e abril de 2019, por meio da observação não participante, tendo como instrumentos dois roteiros de observação. As variáveis descritivas foram analisadas por meio da distribuição de frequência absoluta e relativa. Resultados: destacou-se a ocorrência de 60\% de erros de técnica do preparo e 75\% de erros de técnica de administração dos medicamentos intravenosos. Conclusão: tanto no preparo quanto na administração dos medicamentos intravenosos, foram identificados erros de técnica e de horário, apontando a necessidade de implementação de estratégias voltadas para a segurança do paciente.

Descritores: Erros de Medicação. Administração Intravenosa. Equipe de Enfermagem.

Objective: to identify errors in the preparation and administration of intravenous drugs. Method: observational and descriptive study. 694 doses of intravenous medications performed by nursing professionals in adult patients were observed in the Emergency, Inpatient and Intensive Care Units of a small hospital in the recôncavo of Bahia, Brazil. Data were collected in March and April 2019, through non-participant observation, using two observation scripts as instruments. Descriptive variables were analyzed using the absolute and relative frequency distribution. Results: the occurrence of $60 \%$ of errors in the preparation technique and $75 \%$ of errors in the technique of administering intravenous drugs was bighlighted. Conclusion: both in the preparation and administration of intravenous

\footnotetext{
Enfermeira. Mestra em Enfermagem. Docente da Faculdade de Ciências Empresariais. Santo Antônio de Jesus, Bahia, Brasil. ubianepr@gmail.com. https://orcid. org/0000-0002-5570-8685.

Enfermeira. Doutora em Enfermagem. Docente da Universidade Estadual de Feira de Santana. Feira de Santana, Bahia, Brasil. https://orcid.org/0000-0002-2।045131.

Enfermeiro. Universidade Federal de São Paulo. Docente da Universidade Estadual de Feira de Santana. Feira de Santana, Bahia, Brasil. https://orcid.org/0000-000 I7866-6353.

${ }^{4}$ Fisioterapeuta. Especialista pneumofuncional. Fisioterapeuta intensivista do Instituto de Cardiologia do Recôncavo da Bahia. Santo Antônio de Jesus, Bahia, Brasil. https://orcid.org/0000-0001-5188-1619.

Enfermeira. Especialista em terapia intensiva. Técnica da Universidade Federal do Recôncavo da Bahia. Santo Antônio de Jesus, Bahia, Brasil. https://orcid.org/00000002-5315-7349.

6 Enfermeira. Especialista em terapia intensiva. Enfermeira do Hospital da Mulher Maria Luzia Costa dos Santos. Salvador, Bahia, Brasil. https://orcid.org/0000-000 I-
} $7751-2192$. 
medications, errors in technique and time were identified, pointing out the need to implement strategies aimed at patient safety.

Descriptors: Medication Errors. Administration, Intravenous. Nursing, Team.

Objetivo: identificar errores en la preparación y administración de fármacos intravenosos. Método: estudio observacional y descriptivo. Se observaron 694 dosis de medicamentos intravenosos realizados por profesionales de enfermería en pacientes adultos en las Unidades de Urgencias, Hospitalización y Cuidados Intensivos de un pequeño hospital en el interior de Babia, Brasil. Los datos se recolectaron en marzo y abril de 2019, mediante observación no participante, utilizando dos guiones de observación como instrumentos. Las variables descriptivas se analizaron mediante la distribución de frecuencia absoluta y relativa. Resultados: se destacó la ocurrencia de 60\% de errores en la técnica de preparación y $75 \%$ de errores en la técnica de administración de fármacos intravenosos. Conclusión: tanto en la preparación como en la administración de medicamentos por vía intravenosa se identificaron errores en la técnica y en el tiempo, señalando la necesidad de implementar estrategias orientadas a la seguridad del paciente.

Descriptores: Errores de Medicación. Administración Intravenosa. Grupo de Enfermería.

\section{Introdução}

O erro pode ser definido como uma ocorrência evitável com potencialidade de causar dano desnecessário ao paciente. Evento adverso (EA) é o incidente que resulta em dano ao paciente ${ }^{(1)}$. Estes incidentes, que afetam a segurança do paciente, são considerados um problema de saúde com repercussões internacionais. Nos Estados Unidos, é observado anualmente um elevado número de óbitos, devido a erros e EAs, com taxa de mortalidade maior que aquelas referentes aos acidentes de trânsito, câncer de pulmão ou síndrome da imunodeficiência adquirida ${ }^{(2)}$. O Brasil possui um dos maiores índices de EA evitáveis do mundo ${ }^{(3)}$.

No Brasil, em 2016, a maioria dos eventos notificados relacionados à assistência à saúde, incluindo EA, foram classificados como eventos que causaram danos de grau leve, seguido de nenhum dano. Foram notificados 276 eventos adversos, que resultaram em óbito nesse mesmo ano ${ }^{(4)}$.

O Boletim Segurança do Paciente e Qualidade em Serviços de Saúde disponibiliza ao Sistema Nacional de Vigilância Sanitária (SNVS), por meio do Sistema NOTIVISA, os resultados obtidos da análise dos dados de incidentes relacionados à assistência à saúde notificados pelos Núcleos de Segurança do Paciente (NSP) dos serviços de saúde do Brasil. De acordo com esse boletim, no ano 2016, dentre os óbitos decorrentes de EA relacionados à assistência à saúde notificados, os mais frequentes ocorreram nas categorias classificadas como "outros" e também em "Falhas durante a assistência à saúde", nas quais estão incluídos os erros de medicação. Sendo assim, mesmo com a possibilidade de subnotificação dos eventos adversos no nosso país, os erros de medicação consistem em importantes causas de óbitos dentre os eventos adversos notificados na assistência em saúde ${ }^{(4)}$.

A American Society of Health System Pharmacists (ASHP) define erros de medicação como qualquer evento evitável que pode causar ou levar ao uso inadequado de medicamentos ou dano ao paciente enquanto a medicação está no controle do profissional de saúde, paciente ou consumidor ${ }^{(5)}$.

Recentemente, a Organização Mundial da Saúde lançou o $3^{\circ}$ desafio global intitulado "Medicação sem Dano", visando continuar conscientizando instituições de saúde e governos, pois os erros de medicação ainda ocorrem em grande escala, causando danos aos pacientes ${ }^{(6)}$.

No Brasil, o Programa Nacional de Segurança do Paciente (PNSP), instituído pela Portaria MS/GM no 529, de $1^{\circ}$ de abril de 2013, preconiza a elaboração e implementação de um conjunto de protocolos básicos para minimizar erros e EAs. Entre as prioridades de segurança do paciente, problemas relacionados à prescrição e 
administração de medicamentos merecem destaque, uma vez que são responsáveis pelo maior número de eventos adversos nas instituições de saúde ${ }^{(7)}$.

O erro é considerado, na Teoria do Queijo Suíço, criada por James Reason, como parte do ser humano. Por isso, jamais se conseguirá anular as possibilidades de errar. Entretanto, é possível transformar o ambiente em que os humanos agem, tornando-o mais seguro, com desenhos de sistemas e métodos que evitem os erros, impedindo que perpassem as múltiplas e incompletas camadas de proteção e causem um dano devastador. Sendo assim, os erros na atenção à saúde não são atitudes voluntárias e nem tampouco individuais. Eles são consequências de uma sequência de eventos, não de um único ato isolado, e estão relacionados a um contexto e ao desenvolvimento de um processo assistencial que precisa ser repensado ${ }^{(7-8)}$.

Embora o preparo de medicamentos necessite de conhecimento científico, nas instituições hospitalares, os profissionais de enfermagem costumam executá-lo como tarefa simples e rotineira. Contudo, o manejo inadequado de medicamentos pode diminuir a segurança microbiológica e a eficácia terapêutica, trazendo consequências indesejáveis aos pacientes ou modificando o resultado terapêutico esperado ${ }^{(8)}$. Para garantir esse resultado, devem ser aplicados os princípios científicos no preparo e na administração de medicamentos intravenosos, além de garantir a administração do medicamento certo, considerando também a forma farmacêutica, paciente, horário, via, dose, ação, registro e monitoramento certos ${ }^{(9)}$.

Assim, o preparo e a administração de medicamentos intravenosos é função da equipe de enfermagem que atua na ponta do processo de medicação, sendo a última oportunidade de interceptar a ocorrência de possível erro nesse processo. Entretanto, em um hospital de pequeno porte do recôncavo da Bahia, observou-se baixa frequência de notificação de erros no preparo e na administração de medicamentos intravenosos, indicando possível subnotificação, devido ao fato de que, desde a implantação da ficha de notificação de eventos adversos no ano de 2017, até abril de 2019, foram realizadas apenas nove notificações de erros referentes ao preparo e à administração de medicações.

Tendo em vista que os medicamentos intravenosos oferecem riscos potenciais ao paciente, devido à sua ação rápida na corrente sanguínea, o presente estudo tem como pergunta de investigação: Quais são os erros mais frequentes no preparo e na administração de medicamentos intravenosos num hospital de pequeno porte do recôncavo da Bahia?

Este estudo teve como objetivo identificar os erros no preparo e na administração de medicamentos intravenosos num hospital de pequeno porte do recôncavo da Bahia.

\section{Método}

Trata-se de estudo observacional, descritivo, realizado num hospital privado de pequeno porte localizado na região do Recôncavo da Bahia, que iniciou suas atividades em maio de 2003. Atualmente, essa instituição de saúde dispõe de 50 leitos, distribuídos nos seguintes setores: emergência, internamento, unidade de terapia intensiva (UTI) e centro cirúrgico. Também presta atendimento ambulatorial com especialidades médicas, como ortopedia, urologia, angiologia, clínico geral, cardiologia, gastrologia, neurologia e otorrinolaringologia, assim como exames diagnósticos. A equipe de saúde é composta por Assistente Social, Biomédicos, Enfermeiros, Farmacêuticos, Fisioterapeutas, Médicos, Nutricionistas, Psicólogos e Técnicos de enfermagem. O referido hospital não realiza atendimento de Pediatria e Obstetrícia.

A unidade de análise utilizada na presente pesquisa foram as doses de medicamentos intravenosos preparadas e administradas pela equipe de enfermagem (enfermeiros e técnicos de enfermagem). Adotaram-se os critérios de inclusão: doses de medicamentos intravenosos preparadas e/ou administradas em pacientes hospitalizados nos setores da emergência, UTI e/ou internamento; e doses de medicamentos registradas em prescrição médica. Foram considerados como critérios de exclusão: doses de medicamentos 
prescritas em situações de emergência, medicamentos vasoativos e antiarrítmicos e/ou soluções eletrolíticas, doses de medicamentos preparadas e administradas em pacientes em situações de precauções de contato ou respiratória e em parada cardiorrespiratória.

Não foi incluído o setor do Centro Cirúrgico na coleta de dados, pois $98 \%$ dos medicamentos intravenosos são preparados e administrados pelo anestesista.

$\mathrm{Na}$ presente pesquisa foi utilizada a amostra por conveniência. Para o cálculo do tamanho amostral, a dose preparada e administrada foi considerada como unidade de análise para o erro. Para determinar-se a representatividade amostral de cada setor, foi utilizado o relatório mensal de dispensação de medicamentos da farmácia satélite do referido hospital referente ao mês de dezembro de 2018.

O cálculo amostral foi efetuado, considerando-se o valor de doses por mês nos setores do hospital pesquisado e utilizando-se a fórmula para cálculo amostral de estudos transversais de população finita, um nível de confiança de 95\%, um $\mathcal{E}$ de 0,05 e um valor crítico de 1,96. Considerou-se que podem ser observados $80 \%$ de erros de medicação no preparo e na administração de medicamentos, de acordo com estudo nacional conduzido em uma unidade hospitalar ${ }^{(10-11)}$.

O relatório mensal de dispensação de medicamentos apontou 2.324 doses de medicamentos intravenosos preparados e administrados na UTI, 3.277 doses na emergência e 4.223 doses no internamento. Para a realização da presente pesquisa, o cálculo amostral apontou 223 doses para a UTI, 229 doses para a emergência e 232 doses para o internamento, totalizando uma amostra de 684 doses.

Os dados foram coletados por meio de observação não participante, conduzida por uma equipe de quatro enfermeiras devidamente qualificadas para a aplicação dos instrumentos de pesquisa. O treinamento da equipe teve como objetivo orientá-la quanto à identificação de erros no preparo e na administração de medicamento intravenoso, a maneira de se identificar no momento da coleta junto aos profissionais e ao paciente, a imparcialidade e não interferência nos procedimentos durante a observação, a postura ética durante a abordagem aos profissionais e a qualidade do registro das observações.

A equipe foi treinada em quatro encontros teóricos de quatro horas de duração cada. Nestes, foram abordados os processos de preparo e administração de medicamentos intravenosos, erros de medicação, ética em pesquisa com seres humanos e observação direta do processo de preparo e administração dos medicamentos intravenosos. A seguir, foi realizado um estudo piloto para testagem dos instrumentos de coleta de dados. Cada observador acompanhou o preparo e a administração de três doses. Após cada observação realizada, as dúvidas eram discutidas. O estudo piloto foi realizado durante dois dias no mês de fevereiro, totalizando 12 horas de observação. Não foram realizados ajustes nos itens observados. As observações do estudo piloto não foram incluídas no resultado do estudo.

A coleta de dados foi realizada na emergência, UTI e internamento, nos meses de março e abril de 2019, nos turnos matutino, vespertino e noturno. Foram utilizados dois roteiros de observação - denominados roteiro de observação de preparo de medicamento e roteiro de observação de administração de medicamento - elaborados, validados e autorizados em conjunto com o grupo de pesquisadores de projeto multicêntrico ${ }^{(10-12)}$.

O erro foi considerado como qualquer discrepância identificada entre preparo e/ou administração do medicamento e as diretrizes de preparo e administração do Ministério da Saúde ${ }^{(9)}$. Quando identificada alguma inadequação não indicada no roteiro, o colaborador deveria incluir a descrição no último item do instrumento. O horário foi considerado inadequado, quando foi ultrapassado ou adiantado em 60 minutos do horário prescrito. Utilizou-se como base a definição e a classificação de erros de medicação segundo a $\mathrm{ASHP}^{(5)}$.

Os registros eram realizados nos instrumentos, inicialmente identificando o medicamento preparado e administrado. Em seguida, a preparação e a administração foram conferidas e comparadas com a prescrição médica, observando-se a adequação quanto ao nome do paciente, 
nome do medicamento, dose, via e horário prescrito, bem como quanto à técnica correta. Estes aspectos constavam no roteiro como pontos de observação.

Os dados quantitativos foram validados por dupla digitação em uma planilha eletrônica do programa Excel para Windows. Em seguida, o processamento e a análise das informações foram realizados no programa Statistical Package for the Social Sciences (SPSS) versão 22.0. Foi realizada análise descritiva dos dados coletados, de acordo com as variáveis descritivas selecionadas por meio da distribuição de frequência absoluta e relativa.

O estudo faz parte do projeto de pesquisa intitulado "Segurança no Preparo e Administração de Medicamentos Intravenosos em Pessoas Hospitalizadas" submetido e aprovado pelo Comitê de Ética em Pesquisa com Seres Humanos sob protocolo número 3.153.837. Todas as exigências éticas dispostas nas Resoluções n ${ }^{\circ}$ 466/2012 e $n^{\circ} 510 / 2016$ do Conselho Nacional de Saúde foram integralmente respeitadas.
Cabe salientar que os dados foram coletados após a assinatura do Termo de Consentimento Livre e Esclarecido, garantindo-se o sigilo e a privacidade dos participantes durante todas as fases da pesquisa ${ }^{(13)}$.

\section{Resultados}

Foram observadas 336 doses de preparo de medicamentos intravenosos e 358 de administrações de forma independente e dissociada, totalizando 694 doses. As doses de preparo foram observadas em sua maioria na unidade de internamento. Já as de administração, foram observadas na UTI. Quanto ao turno de trabalho, foram observadas as doses de preparo em quantidade maior no turno vespertino; as doses de administração, no turno noturno. Tanto o preparo quanto a administração foram majoritariamente realizados por técnicos de enfermagem das unidades pesquisadas (Tabela 1).

Tabela 1 - Distribuição do número de doses de medicamentos preparados e administrados em adultos hospitalizados conforme unidade hospitalar, categoria profissional e turno de trabalho. Santo Antônio de Jesus, Bahia, Brasil - 2020

\begin{tabular}{|c|c|c|}
\hline Variáveis & $\mathbf{n}$ & $\%$ \\
\hline \multicolumn{3}{|c|}{ Unidade na qual foram observadas as doses do preparo } \\
\hline Emergência & 108 & 32 \\
\hline UTI & 105 & 31 \\
\hline Internamento & 123 & 37 \\
\hline \multicolumn{3}{|c|}{ Unidade na qual foram observadas as doses de administração } \\
\hline Emergência & 107 & 30 \\
\hline UTI & 133 & 37 \\
\hline Internamento & 118 & 33 \\
\hline \multicolumn{3}{|c|}{ Profissional responsável pelo preparo das doses } \\
\hline Técnico de enfermagem & 277 & 82 \\
\hline Enfermeiro & 59 & 18 \\
\hline \multicolumn{3}{|c|}{ Profissional responsável pelas doses administradas } \\
\hline Técnico de enfermagem & 286 & 80 \\
\hline Enfermeiro & 72 & 20 \\
\hline \multicolumn{3}{|c|}{ Turno de trabalho no qual as doses observadas foram preparadas } \\
\hline Matutino & 108 & 32 \\
\hline Vespertino & 117 & 35 \\
\hline Noturno & 111 & 33 \\
\hline \multicolumn{3}{|c|}{ Turno de trabalho no qual as doses observadas foram administradas } \\
\hline Matutino & 109 & 30 \\
\hline Vespertino & 122 & 34 \\
\hline Noturno & 127 & 36 \\
\hline
\end{tabular}

Fonte: Elaboração própria. 
Durante a observação, foram identificados erros no preparo das doses de medicamentos intravenosos, sendo 60\% relacionados à técnica. Não foram identificados erros de dose, via e/ou paciente. Dentre os tipos de erros de técnica no preparo dos medicamentos intravenosos, destacaram-se: não utilização de equipamentos de proteção individual (EPIs) e não identificação do medicamento preparado. Na tabela, é possível observar elevado percentual de ausência da higienização das mãos antes do preparo, da desinfecção das ampolas e da desinfecção da bancada (Tabela 2).

Tabela 2-Distribuição da tipologia dos erros no preparo e na administração das doses de medicamentos intravenosos em adultos hospitalizados. Santo Antônio de Jesus, Bahia, Brasil - 2020

\begin{tabular}{|c|c|c|c|c|}
\hline \multirow{2}{*}{ Tipos de erros observados } & \multicolumn{2}{|c|}{ Não } & \multicolumn{2}{|c|}{ Sim } \\
\hline & $\mathbf{n}$ & $\%$ & $\mathbf{n}$ & $\%$ \\
\hline \multicolumn{5}{|l|}{ Tipos de erros no preparo } \\
\hline Técnica & 203 & 60 & 133 & 40 \\
\hline Horário & 8 & 2 & 328 & 98 \\
\hline Dose & - & - & 336 & 100 \\
\hline Via & - & - & 336 & 100 \\
\hline Paciente & - & - & 336 & 100 \\
\hline \multicolumn{5}{|l|}{ Tipos de erros na técnica de preparo das doses (1) } \\
\hline Uso de prescrição médica & 321 & 95 & 15 & 5 \\
\hline Desinfecção da bancada & 267 & 80 & 69 & 20 \\
\hline Desinfecção de ampolas & 219 & 65 & 117 & 35 \\
\hline Higienização das mãos & 185 & 55 & 151 & 45 \\
\hline Identificação correta do medicamento preparado & 159 & 47 & 177 & 53 \\
\hline Utilização de EPIs & 128 & 38 & 208 & 62 \\
\hline Contaminação de material & 3 & 1 & 333 & 99 \\
\hline \multicolumn{5}{|l|}{ Tipos de erros na administração } \\
\hline Técnica & 268 & 75 & 90 & 25 \\
\hline Horário & 12 & 3 & 346 & 97 \\
\hline Dose & - & - & 358 & 100 \\
\hline $\mathrm{Via}$ & - & - & 358 & 100 \\
\hline Paciente & - & - & 358 & 100 \\
\hline \multicolumn{5}{|l|}{ Tipos de erros na técnica de administração (1) } \\
\hline Uso de prescrição médica & 213 & 60 & 145 & 40 \\
\hline Identificou o paciente & 188 & 52 & 170 & 48 \\
\hline Orientou o paciente & 256 & 71 & 102 & 29 \\
\hline Higienização das mãos & 148 & 41 & 210 & 59 \\
\hline Conferência com prescrição ou rótulo & 216 & 60 & 142 & 40 \\
\hline Utilização de EPIs & 178 & 50 & 180 & 50 \\
\hline $\begin{array}{l}\text { Desinfecção da ponta do dispositivo do acesso } \\
\text { intravenoso }\end{array}$ & 119 & 33 & 239 & 67 \\
\hline Contaminação de material & 7 & 2 & 351 & 98 \\
\hline \multicolumn{5}{|l|}{ Tipos de erros na técnica de administração (1) } \\
\hline Retornou para monitoramento & 212 & 59 & 146 & 41 \\
\hline Checagem da administração & 313 & 87 & 45 & 13 \\
\hline
\end{tabular}

Fonte: Elaboração própria.

Notas: Sinais convencionais utilizados:

- Dado numérico igual a zero não resultante de arredondamento.

(1) Uma dose observada apresentou diversos tipos de erros.

No que se refere à observação das doses administradas, o maior percentual de erros foi relacionado à técnica. Não foram identificados erros de dose, via e/ou paciente durante a observação da administração das medicações (Tabela 2). Dentre os tipos de erros de técnica na 
administração das doses de medicamentos intravenosos, destacaram-se: ausência da desinfecção da ponta do dispositivo do acesso intravenoso, não higienização das mãos e não identificação do paciente.

\section{Discussão}

Erros no preparo e na administração de medicamentos constituem um problema de saúde pública devido à sua ocorrência, principalmente quando se refere a medicamentos intravenosos. Neste caso, o manuseio inadequado pode acarretar eventos adversos nos pacientes, devido à sua ação na corrente sanguínea. Tal condição impõe que essa prática seja exercida de forma correta e segura, para que os erros sejam minimizados e prevenidos $^{(14)}$.

O fato de técnico de enfermagem ter sido a categoria profissional mais observada neste estudo está de acordo com achados da literatura nacional $^{(14-15)}$. Ademais, a responsabilidade compartilhada do enfermeiro e do técnico de enfermagem na administração de medicamento contempla a Resolução n ${ }^{\circ}$ 564/2017 do Conselho Federal de Enfermagem (COFEN) ${ }^{(16)}$.

Contudo, de acordo com o COFEN, no Brasil, existem 1.250.953 técnicos de enfermagem, 536.325 enfermeiros e 413.952 auxiliares de enfermagem $^{(17)}$. Estes dados informam que o número de técnicos de enfermagem é superior ao dobro do número de enfermeiros. Estes, em sua prática clínica diária, devido à elevada carga de trabalho, tendem a transferir a responsabilidade do preparo e da administração de medicamentos intravenosos para os profissionais de nível médio, afastando-se de cuidado direto ao paciente e priorizando ações gerenciais nas unidades.

Entretanto, o erro de medicação desencadeia impactos variáveis no quadro de saúde do paciente, podendo resultar em morte ou complicações no quadro clínico, com prolongamento da internação hospitalar, o que gera dano físico ao paciente e encargos para a instituição ${ }^{(18)}$.

O erro de técnica foi identificado em 60\% das doses preparadas. Em estudo realizado na Alemanha, o índice de $56 \%{ }^{(19)}$ foi identificado.
Estudos ${ }^{(8,14-15,18,20)}$ com população adulta apresentaram resultados compatíveis. Entretanto, estudo $^{(21)}$ realizado no Vietnã revelou índice bem inferior $(15,7 \%)$.

Nesta pesquisa, os principais tipos de erros observados na técnica de preparo dos medicamentos intravenosos foram a não utilização de EPIs (62\%), identificação incorreta do medicamento preparado (53\%), falta de higienização das mãos (45\%), não desinfecção de ampolas (35\%) e não desinfecção da bancada (20\%). Estes são também os principais tipos de erros observados em estudos nacionais ${ }^{(8,14-15,18,20)}$.

A realização das medicações intravenosas com qualidade, segurança e eficácia requer o cumprimento de requisitos mínimos, para garantir a total ausência de contaminações químicas e biológicas ${ }^{(13)}$. A segurança microbiológica pode estar diminuída, quando os profissionais de enfermagem não realizam a higiene das mãos, não limpam a bancada antes de preparar os medicamentos, não usam máscara para preparar as soluções e não realizam a desinfecção de ampolas ${ }^{(8-9)}$.

O risco de contaminação na remoção da dose do medicamento de frasco-ampola está relacionado ao tipo de frasco, características da agulha ou outro dispositivo de punção empregado para remover a dose, número de perfurações na borracha, características físicas da borracha, técnica asséptica utilizada, injeção de ar no frasco e eficiência dos conservantes ${ }^{(8-9,13)}$.

A higienização das mãos não foi realizada de forma correta. Nas desinfecções das ampolas, foi utilizado algodão não estéril embebido em álcool a 70\%, sem movimento de fricção mecânica e não aguardando 15 segundos para secar a solução. Estes achados também estavam presentes em outros estudos descritivos de observação de erros no preparo e na administração de medicamentos ${ }^{(15)}$.

Durante o preparo das medicações, observou-se a não desinfecção da bancada em 20\% das doses. Ignorou-se que superfícies limpas e desinfetadas conseguem reduzir em cerca de $90 \%$ o número de microrganismos, enquanto as superfícies que foram apenas limpas reduzem-nos em 
80\%, por apenas duas horas. Portanto, indica-se armazenar, acessar e preparar medicamentos e materiais/suprimentos em uma área ou superfície limpa de acordo com o manual de limpeza e desinfecção da Agência Nacional de Vigilância Sanitária (ANVISA) ${ }^{(8,22)}$.

Devem ser seguidas as Medidas de Prevenção de Infecção Relacionada à Assistência à Saúde preconizadas pela ANVISA, quanto ao preparo e à administração de medicamentos, atentando-se para a desinfecção do ambiente e de superfícies, higienização das mãos, uso de equipamentos de proteção, desinfecção de ampolas, frascos, pontos de adição dos medicamentos e conexões das linhas de infusão ${ }^{(23)}$.

A não higiene das mãos, o não uso de EPIs (máscaras, luvas e touca), não desinfecção de ampolas e não desinfecção da ponta do dispositivo do acesso intravenoso acarretam translocação bacteriana para o sítio de infusão da dose e pele do paciente, aumentando o risco de flebite infecciosa e infecção de corrente sanguínea relacionada ao cateter. Essas medidas individuais são simples, pouco dispendiosas e previnem a propagação das infecções relacionadas à assistência à saúde.

Quanto à eficácia terapêutica, pode estar reduzida quando não se garante a estabilidade química e física dos medicamentos intravenosos, o que pode comprometer o efeito terapêutico do medicamento. Portanto, ao se reconstituir ou diluir medicamentos, deve-se utilizar o diluente adequado, de acordo com o Protocolo de Diluição, considerar a hora do preparo e verificar o prazo de estabilidade após a reconstituição/diluição ${ }^{(8-9,13)}$.

A ausência de protocolo institucional direcionado ao preparo de medicamentos intravenosos pode ter favorecido os erros da técnica identificados durante a preparação, destacando-se a ausência da rotulagem dos medicamentos em bolus. Este fato pode induzir ao erro de medicação, pois, quando o profissional deixa de realizar a rotulagem do medicamento, desfavorece a realização da tripla checagem antes da administração.

No que se refere à observação da administração das doses de medicamentos, foram identificados 75\% de erros de técnica de administração. Estudos nacionais apresentaram resultados similares ${ }^{(14,18,20)}$. Entretanto, estudo internacional multicêntrico, realizado na Inglaterra, revelou índice bem inferior $(11,5 \%)^{(24)}$.

Quanto à tipologia dos erros de técnica de administração, destacaram-se: ausência de higiene das mãos antes (59\%), desinfecção da ponta do dispositivo do acesso intravenoso (67\%), utilização de EPIs (50\%), checagem da prescrição ou rótulo (40\%), identificação (48\%), monitoramento (41\%) e orientação do paciente (29\%). Estes achados assemelham-se aos de estudos nacionais ${ }^{(14,18,20)}$.

Para uma prática segura na administração de medicamentos, é necessário conhecimento de todos os aspectos relacionados à medicação, como a ação, indicação, contraindicação, efeitos colaterais e interações indesejáveis no organismo, formas de preparo, modo e via corretos de sua administração. É igualmente necessário que se dedique atenção na realização desse procedimento, de modo a proporcionar ações seguras a todos os envolvidos no processo, ao garantir a administração do medicamento certo, na forma farmacêutica certa, ao paciente certo, na hora certa, na via certa, na dose correta, com ação certa, com registro certo e com monitoramento certo ${ }^{(13)}$.

No presente estudo, foram observados erros de horário no preparo do medicamento em 2\% das doses observadas e na administração deste em 3\% das doses. Pesquisas nacionais corroboraram o achado, ao observarem erros de via, dose e horário ${ }^{(14,20,25)}$.

O preparo e a administração tendem a ser realizados em horário incorreto, devido à prática frequente de otimizar ou adiantar as atividades, e ainda devido à sobrecarga de trabalho. Entretanto, devem ser evitados por meio de estratégias e da supervisão ativa, já que medicamentos podem ter comprometimento de sua estabilidade quando diluídos muito precocemente e não administrados, além de estarem expostos a contaminação, luz, calor e umidade. Outro fator relevante é o tempo e a duração da ação dos medicamentos no organismo, uma vez 
que a administração fora do horário previsto pode diminuir essa ação devido à meia-vida do preparado, comprometendo a recuperação do paciente ${ }^{(15)}$.

Não foram observados erros de dose, de via e de troca de paciente e/ou medicamento, resultado que concorda com o encontrado em estudo $^{(18)}$ no qual tais eventos não ocorreram durante o período de observação.

Para instituições que buscam a qualidade do serviço e a segurança do paciente, uma importante estratégia para a redução de erros de medicação consiste em investir no conhecimento dos profissionais de enfermagem sobre esse assunto, de modo a envolvê-los na segurança medicamentosa, minimizando os fatores que ocasionariam o erro. Aliado a isso, a inclusão de estratégias para reduzir os erros humanos, como passo a passo visível no local de preparo e administração do medicamento, podem contribuir para diminuir os lapsos de memória e as inúmeras interrupções que o profissional sofre no momento em que prepara o medicamento, além de reduzir a probabilidade de erros e aumentar a chance de interceptá-los antes de resultarem em prejuízo ao paciente ${ }^{(8)}$.

O estudo teve como limitação ter trabalhado com amostra por conveniência, havendo, assim, turnos e setores com maior número de observações. Apesar dessas limitações, o estudo traz avanços para a enfermagem, na medida em que contribui com evidências sobre o preparo e a administração de medicamentos pela equipe de enfermagem. Pode contribuir também na condução de novos estudos sobre o tema em diferentes contextos.

\section{Conclusão}

Com o presente estudo pôde-se concluir que erros de técnica e de horário no preparo e na administração dos medicamentos intravenosos ocorreram no hospital de pequeno porte do recôncavo da Bahia. Os principais tipos de erros de técnica observados no preparo foram a não utilização de EPIs, a identificação incorreta do medicamento preparado, a falta de higiene das mãos, a não desinfecção de ampolas e a não desinfecção da bancada. Dentre os erros de técnica observados na administração, destacaram-se a ausência de higiene das mãos antes da administração dos medicamentos e de desinfecção da ponta do dispositivo do acesso intravenoso, a não utilização de EPIs, a não checagem da prescrição ou rótulo, não identificação, não monitoramento e não orientação do paciente.

\section{Colaborações:}

1 - concepção, projeto, análise e interpretação dos dados: Ubiane Oiticica Porto Reis, Silvia da Silva Santos Passos, Jamille Sampaio Berhends e Carolina Madeiro Meira;

2 - redação do artigo e revisão crítica relevante do conteúdo intelectual: Ubiane Oiticica Porto Reis, Silvia da Silva Santos Passos, Luciano Marques Santos e Marcelo Silva Reis;

3 - aprovação final da versão a ser publicada: Ubiane Oiticica Porto Reis e Silvia da Silva Santos Passos.

\section{Referências}

1. World Health Organization. A World Alliance for Safer Health Care. More Than Words: Conceptual Framework for the International Classification for Patient Safety. Version 1.1. Final Technical Report [Internet]. Geneva (CH); 2009 [cited 2020 Jan 10]. Available from: http://www.who.int/patientsafety/ taxonomy/icps_full_report.pdf

2. Kohn LT, Corrignan JM, Donaldson MS. To err is human: building a safer health system. Washington (DC): National Academy Press; 2000.

3. Menegueti MG, Garbin LM, Oliveira MP, Shimura CMN, Guilherme C, Rodrigues RAP. Errors in the medication process: proposal of an education strategy based on notified errors. J Nurs UFPE on line. 2017;11(Supl. 5):2046-55. DOI: https://doi.org/10.5205/1981-8963v11i5a23358p2046-2055-2017

4. Brasil. Agência Nacional de Vigilância Sanitária. Boletins Informativos: Segurança do paciente e qualidade em serviços de saúde [Internet]. Brasília (DF); 2017 [cited 2020 Jan 10]. Available from: http://portal.anvisa.gov.br/ documents $/ 33852 / 271855 / \%$ C3\%A7a + do $+P a c$ 
iente+e+Qualidade+em+Servi\%C3\%A7os+de+ Sa\%C3\%BAde +n\%C2\%BA+15/bb6373924973-4e7f-8907-a7b3af1e297b

5. American Society of Health-System Pharmacists. ASHP guidelines on preventing medication errors in hospitals. Am J Health Syst Pharm. 2018;75(19):1493-517. DOI: 10.2146/ajhp170811

6. World Health Organization. Medication Without Harm - Global Patient Safety Challenge on Medication Safety [Internet]. Geneva (CH); 2017 [cited 2020 Jan 10]. Available from: http://apps. who.int/iris/bitstream/10665/255263/1/WHO-HISSDS-2017.6-eng.pdf?ua $=1 \& u a=1$

7. Brasil. Ministério da Saúde. Fundação Oswaldo Cruz; Agência Nacional de Vigilância Sanitária. Documento de referência para o Programa Nacional de Segurança do Paciente [Internet]. Brasília (DF); 2014 [cited 2020 Jan 10]. Available from: https://proqualis.net/sites/proqualis.net/ files/documento_referencia_programa_nacional_ seguranca.pdf

8. Camerini FG, Silva LD. Segurança do paciente: análise do preparo de medicação intravenosa em hospital da rede sentinela. Texto Contexto Enferm. 2011;20(1):41-9. DOI: https://doi.org/10.1590/ S0104-07072011000100005

9. Brasil. Ministério da Saúde. Agência Nacional de Vigilância Sanitária. Protocolo de segurança na prescrição, uso e administração de medicamentos [Internet]. Brasília (DF); 2013 [cited 2020 Jan 12]. Available from: https://www20.anvisa.gov.br/ segurancadopaciente/index.php/publicacoes/item/ seguranca-na-prescricao-uso-e-administracao-demedicamentos

10. Opitz SP. Sistema de medicação: análise dos erros nos processos de preparo e administração de medicamentos em um hospital de ensino [Internet]. [tese]. Ribeirão Preto (SP): Universidade de São Paulo; 2006 [cited 2020 Jan 8]. Available from: https://www.teses.usp.br/teses/ disponiveis/22/22132/tde-11092008-163213/ publico/simoneperufoopitz.pdf

11. Triola MF. Introdução à Estatística. 7a ed. Rio de Janeiro: LTC; 1999.

12. Cassiani SHB, Opitz SP, Silva AEBC, Fakih FT, Teixeira TCA, Mota MLS, et al. Identificação e análise dos erros de medicação em hospitais brasileiros. Ribeirão Preto: EERP/USP; 2006.

13. Brasil. Ministério da Saúde. Conselho Nacional de Saúde. Resolução n 510, de 7 de abril de 2016.
Dispõe sobre as normas aplicáveis a pesquisas em Ciências Humanas e Sociais cujos procedimentos metodológicos envolvam a utilização de dados diretamente obtidos com os participantes ou de informações identificáveis ou que possam acarretar riscos maiores do que os existentes na vida cotidiana [Internet]. Brasília (DF); 2016 [cited 2020 Jan 12]. Available from: https://bvsms.saude.gov. br/bvs/saudelegis/cns/2016/res0510_07_04_2016. html

14. Pereira FGF, Aquino GÂ, Melo GAA, Praxedes CO, Caetano JÁ. Conformidades e não conformidades no preparo e administração de antibacterianos. Cogitare Enferm. 2016;21(5):1-9. DOI: http:// dx.doi.org/10.5380/ce.v21i5.45506

15. Mendes JR, Lopes MCBT, Campanharo CRV, Okuno MFP, Batista REA. Tipos e frequência de erros no preparo e na administração de medicamentos endovenosos. einstein (São Paulo); 2018;16(3):eA04146. DOI: https://doi.org/10.1590/ s1679-45082018ao4146

16. Brasil. Conselho Federal de Enfermagem. Resolução COFEN n 564, de 6 de novembro de 2017. Aprova o novo Código de Ética dos Profissionais de Enfermagem [Internet]. Brasília (DF); 2017 [cited 2020 Jan 11]. Available from: http://www.cofen. gov.br/resolucao-cofen-no-5642017_59145.html

17. Brasil. Conselho Federal de Enfermagem. Enfermagem em números [Internet]. Brasília (DF); 2019 [cited 2020 Jan 11]. Available from: http:// www.cofen.gov.br/enfermagem-em-numeros

18. Lemos NRF, Silva VR, Martinez MR. Fatores que predispõem à distração da equipe de enfermagem durante o preparo e a administração de medicamentos. REME rev min enferm [Internet]. 2012 [cited 2020 Jan 11];16(2):201-7. Available from: https://pesquisa.bvsalud.org/portal/resource/pt/ lil-653226

19. Hermanspann T, van der Linden E, Schoberer M, Fitzner C, Orlikowsky T, Marx G, et al. Evaluation to improve the quality of medication preparation and administration in pediatric and adult intensive care units. Drug Healthc Patient Saf. 2019;11:11-8. DOI: $10.2147 /$ DHPS.S184479

20. Santana BS, Rodrigues BS, Stival MM, Rehem TCMSB, Lima LR, Volpe CRG. Interrupções no trabalho da enfermagem como fator de risco para erros de medicação. Av Enferm. 2019;37(1):56-64. DOI: https://doi.org/10.15446/av.enferm.v37n1.71178 
21. Nguyen H-T, Nguyen T-D, Van den Heuvel ER, Haaijer-Ruskamp FM, Taxis K. Medication Errors in Vietnamese Hospitals: Prevalence, Potential Outcome and Associated Factors. PLoS One. 2015;10(9). DOI: 10.1371/journal.pone.0138284

22. Brasil. Agência Nacional de Vigilância Sanitária. Segurança do paciente em serviços de saúde: limpeza e desinfecção de superfícies [Internet]. Brasília (DF); 2012 [cited 2020 Jan 18]. Available from: file://C:/Users/Marcelo/Downloads/Manual_ Limpeza_e_Desinfeccao_final.pdf

23. Brasil. Agência Nacional de Vigilância Sanitária. Medidas de Prevenção de Infecção Relacionada à Assistência à Saúde [Internet]. Brasília (DF); 2017 [cited 2020 Jan 18]. Available from: http:// portal.anvisa.gov.br/documents/33852/3507912/ Caderno+4+-+Medidas+de+Preven $\% \mathrm{C} 3 \% \mathrm{~A} 7 \% \mathrm{C} 3$ $\% \mathrm{~A} 3 \mathrm{O}+\mathrm{de}+\mathrm{Infec} \% \mathrm{C} 3 \% \mathrm{~A} 7 \% \mathrm{C} 3 \% \mathrm{~A} 3 \mathrm{O}+$ Relacionada+
$\% \mathrm{C} 3 \% \mathrm{~A} 0+$ Assist $\% \mathrm{C} 3 \% \mathrm{AAncia}+\% \mathrm{C} 3 \% \mathrm{~A} 0+\mathrm{Sa} \% \mathrm{C} 3 \%$ BAde/a3f23dfb-2c54-4e64-881c-fccf9220c373

24. Lyons I, Furniss D, Blandford A, Chumbley G, Iacovides I, Wei L, et al. Errors and discrepancies in the administration of intravenous infusions: a mixed methods multihospital observational study. BMJ Qual Saf. 2018;27(11):892-901. DOI: 10.1136/bmjqs-2017-007476

25. Llapa-Rodriguez EO, Silva LSL, Menezes MO, Oliveira JKA, Currie LM. Assistência segura ao paciente no preparo e administração de medicamentos. Rev Gaúcha Enferm. 2017;38(4):e2017-0029. DOI: https://doi.org/ 10.1590/1983-1447.2017.04.2017-0029

Recebido: 7 de maio de 2020

Aprovado: 30 de junho de 2020

Publicado: 16 de outubro de 2020

A Revista Baiana de Enfermagem utiliza a Licença Creative Commons - Atribuição-NãoComercial 4.0 Internacional.

https://creativecommons.org/licenses/by-nc/4.0/ Este artigo é de acesso aberto distribuído sob os termos da Licença Creative Commons (CC BY-NC). Esta licença permite que outros remixem, adaptem e criem a partir do seu trabalho para fins não comerciais. Embora os novos trabalhos tenham de lhe atribuir o devido crédito e não possam ser usados para fins comerciais, os usuários não têm de licenciar esses trabalhos derivados sob os mesmos termos. 